\title{
Rf Propagation Through Transparent Conductors In Energy Efficient Windows
}

\author{
D. Stolhofer, H. Doelecke \\ Elektro- und Informationstechnik \\ Fachhochschule Hannover \\ Hannover, Germany
}

\author{
Yaqiang Liu, P.O'Leary \\ Engineering Technology \\ Waterford Institute of Technology \\ Waterford, Ireland
}

\begin{abstract}
Windows were traditionally used as a means of building access and egress for RF signals. However, the drive towards building energy efficiency now means that windows are frequently coated with thin layers of Transparent Conductors (TCs). TCs can let visible light energy pass virtually unattenuated, but reflect longer wavelengths (typically from the infrared region), to keep buildings warmer in colder climates and cooler in warmer climates. However, the use of TCs has a negative impact on wireless propagation, which this paper reports on. Three commercially available windows are examined, with results showing that less than $1 \%$ of the signal passes in either direction. This result is taken from a series of measurement over a range of frequencies from $800 \mathrm{MHz}$ to 6 GHz, both in a large hall and in the WIT anechoic chamber.
\end{abstract}

Keywords-microwave; propagation; reflection coefficient; energy efficient glass;

\section{INTRODUCTION}

Transparent conductors (TCs) are gaining widespread acceptance in glass manufacture, as a means of controlling the energy leaving (or entering in warmer climates) buildings. Legislation has been introduced to encourage this, such as Germany's Verordnung ueber energiesparenden Waermeschutz und energie sparende Anlagentechnik bei Gebaeuden (Energiesparverordnug - EneV), which states that the energy demand shall be reduced by over $30 \%$ and in a further step by the year 2012 by a further $30 \%$, compared with previous legislated levels [1].

The industry response has focused primarily on the use of TC coatings, usually on one of the inward facing sides in a double glazed unit. The aim of applying thin coats of TC is to reduce heat transfer across a vertically mounted window from about $3-1.5 \mathrm{Wm}^{-2} \mathrm{~K}^{-1}$ to as low as $0.4 \mathrm{Wm}^{-2} \mathrm{~K}^{-1}$ for double glazing, principally by reducing the radiative energy loss for frequencies beyond (lower than) the visible part of the spectrum [2]. The challenge for window manufacturers, in using TCs, is to also maintain light transmittance in the visible part of the electromagnetic spectrum, as shown in the $\mathrm{TiO} 2 / \mathrm{Ag} / \mathrm{TiO} 2$ coated glass graph in Figure 1 (reproduced from [2]).

Quite a lot of research has gone into the development and testing of various TCs, but mainly focusing on performance in the visible and near Infra-red regions of the spectrum. This research examines the knock-on effect for Radio Frequency (RF) propagation.

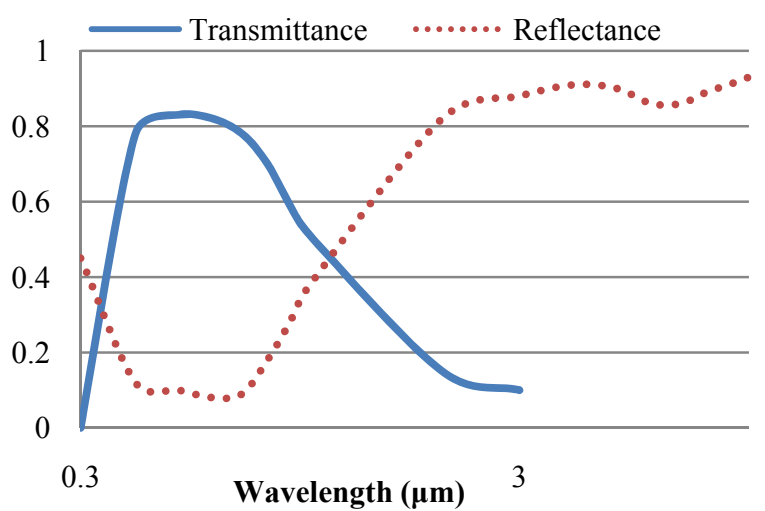

Figure1. $\mathrm{TiO} 2 / \mathrm{Ag} / \mathrm{TiO} 2$ coating transmittance and reflectance (reproduced from [2])

Although published TC data measurements typically stop at about wavelengths of $3 \mu \mathrm{m}$ and the work here covers RF wavelengths from 5 to $37.5 \mathrm{~cm}$, it may be expected from the published graphs that the RF propagation would be quite low.

This large expected loss due to the coating may be contrasted with the low expected loss in passing through the silica float glass alone, as reported in [3] for the range 0.5$2 \mathrm{GHz}$ and reproduced here in Figure 2. The glass reported on here is $4 \mathrm{~mm}$ thick (single pane), twice $6 \mathrm{~mm}$ (coated double glazed unit- Glass A) and twice $4 \mathrm{~mm}$ (coated double glazed unit- Glass B).

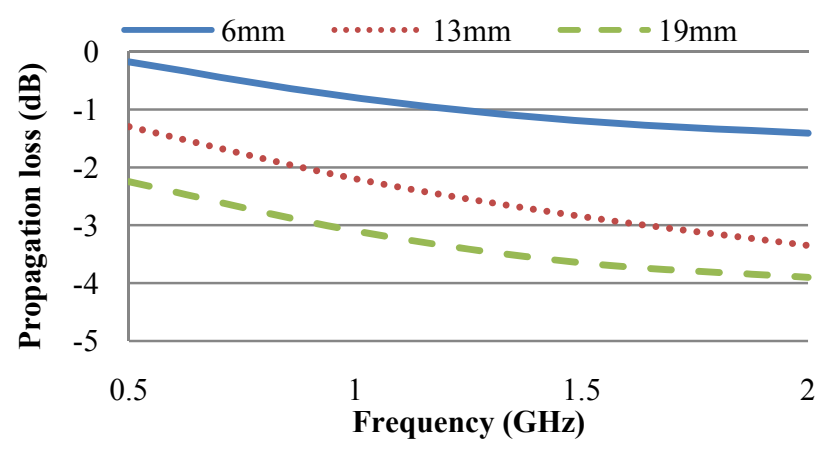

Figure2. Glass propagation measurements from [3] 
From the graphs, it could be expected that the losses up to $2 \mathrm{GHz}$ passing through various glass thicknesses would be less than $1 \mathrm{~dB}$ for $4 \mathrm{~mm}$, less than $2.5 \mathrm{~dB}$ for $8 \mathrm{~mm}$ and less than $3.5 \mathrm{~dB}$ for $12 \mathrm{~mm}$. In fact the measurement results presented here show that the coating can be the dominant mechanism in RF propagation through glasses, reducing the signal by as much as $30 \mathrm{~dB}$.

\section{MEASUREMENT METHOD}

\section{A. Measurement Set-up}

The tests were initially performed in a big hall (approximately $45 \mathrm{~m} \times 30 \mathrm{~m} \times 8 \mathrm{~m}$ ). The propagation properties are measured in the far field, which is defined, at the test frequencies $(800 \mathrm{MHz}-6 \mathrm{GHz})$, and by a combination of $3 \lambda, 5 \mathrm{D}$ and $2 \cdot D^{2} / \lambda$, (where $D$ is the biggest antenna dimension and $\lambda$ is the test wavelength). For the chosen antennas (Schwarzbeck horn antenna BBHA 9120 LFA), $\mathrm{D}=0.33 \mathrm{~m}$. Thus the minimum far field separation, at the maximum test frequency of $6 \mathrm{GHz}$, is $4.35 \mathrm{~m}$, which we round up to $5 \mathrm{~m}$. Measurements were also taken at $7 \mathrm{~m}$ separation and, over a narrower, lower range of frequencies, at a separation of $1.65 \mathrm{~m}$. The antennas were mounted on a modified Pentax Precision Total Station (Figure 3), which permitted accurate distance and angular measurements.
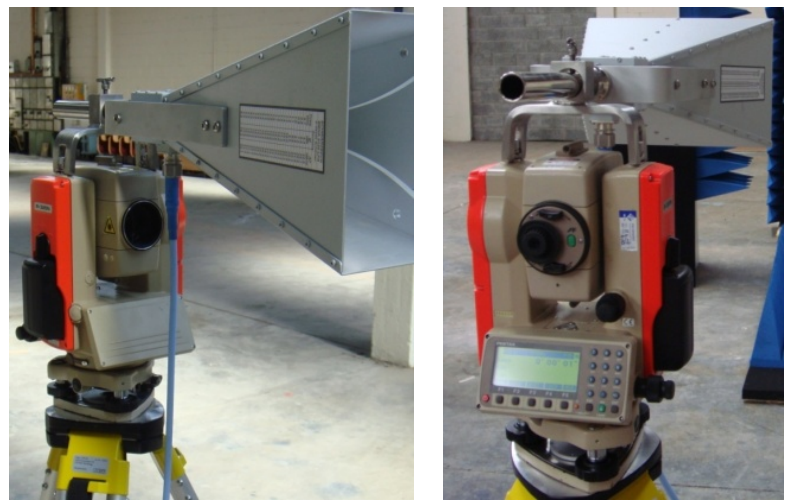

Figure3. Modified Total Station Antenna Mount

The measurements were performed with a Rohde \& Schwarz ZVB-20 Vector Network Analyser (VNA). The Rhode \& Schwarz calibration kit ZV-Z32 was used for the cable calibrations. The cables chosen were $7.62 \mathrm{~m}$ long Sucoflex 106, low-loss cables from Huber + Suhner.

Figure 4 shows the test configuration for the large hall. To reduce the influence of unwanted ground, rear or side wall reflections, the receiver was surrounded by absorbing foam (45cm deep Siepel APM45, with minimum normal incidence reflectivity of -30 to $-50 \mathrm{~dB}$ over the measurement range). The antenna height above the floor was $1.5 \mathrm{~m}$, to reduce the ground reflection influence, against which effect absorbent foam was also laid on the concrete floor. Measurement results were later confirmed in our anechoic chamber, albeit from 1 to $2 \mathrm{GHz}$ only, due to smaller chamber dimensions (approximately $3 \mathrm{~m} \mathrm{x}$ $2.9 \mathrm{~m} \times 2 \mathrm{~m}$ ) and the far field criteria.

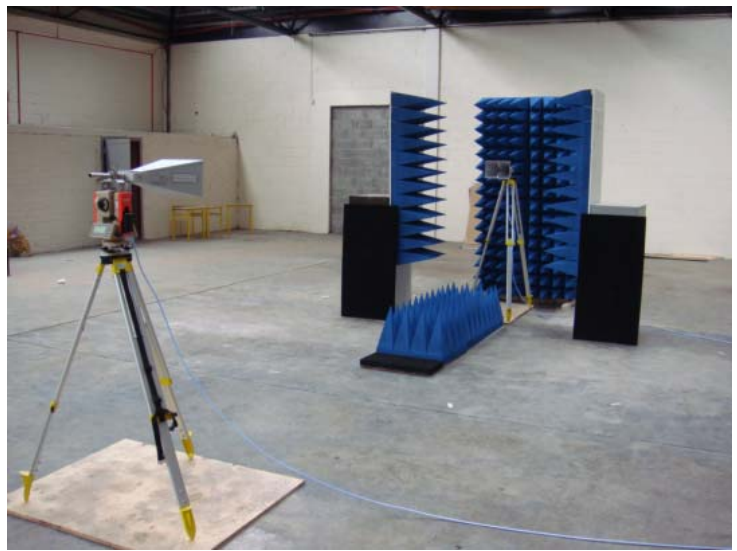

Figure4. Large hall test configuration, without wooden frame

A custom-built wooden frame $(2.1 \mathrm{~m} \times 1.1 \mathrm{~m}$ cross-section $)$ with rounded edges (Figure 5) was created to hold the glass under test in a position normal to the propagating wave.
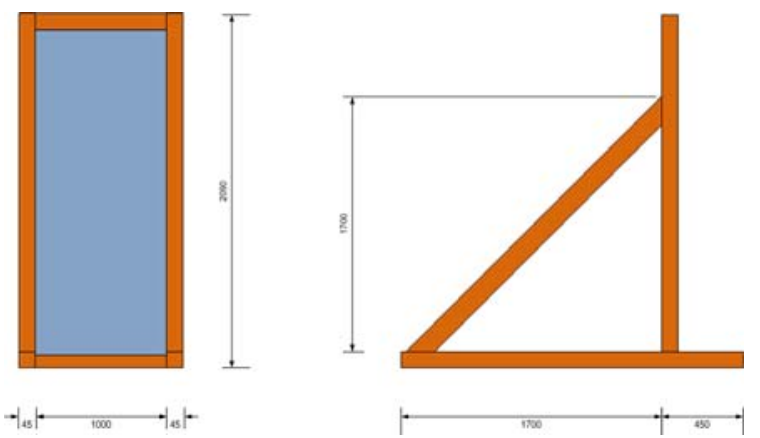

Figure5. Custom-built test frame

\section{B. Measurement Procedure}

Two double-glazed glasses were measured, one identified here as Coated Glass Type A, with dimensions $(2 \mathrm{~m} \times 1 \mathrm{~m})$ and the other one identified here as Coated Glass Type B $(0.9 \mathrm{~m} x$ $0.45 \mathrm{~m})$. The glass and cavity thicknesses were $6 \mathrm{~mm} \& 12 \mathrm{~mm}$ respectively for the Coated Glass A and $4 \mathrm{~mm} \& 8 \mathrm{~mm}$ respectively for the Coated Glass B. As mentioned in the Introduction, a sheet of $4 \mathrm{~mm}$ thick plain glass with the dimensions of $0.89 \mathrm{~m}$ by $1.37 \mathrm{~m}$ was also tested for comparison purposes.

As a VNA was used for the propagation measurements, it was possible to easily examine the effect of the glass under test alone, by doing a two-port one-path calibration. To do this both transmit and receive antennas stood in the position, which is selected for the "normal" measurement, with only the glass under test missing from the configuration. The results for $S_{21}$ and $S_{12}$ were stored in the VNA, as baseline data. This fundamental data was later used to remove the effects of the cables, antenna, etc, in a manner similar to a standard VNA calibration. The $S_{21}$ and $S_{12}$ were then measured with the glass under test in place.

Each of the glasses was tested for RF propagation as follows. The antennas were placed at a separation defined by 
the Total Station, with each glass held in the test frame, which was placed just in front of the receiver antenna.

Results presented here are for E-plane measurements. Hplane measurements are not presented, although the results are similar.

\section{RESULTS}

Energy efficient windows must first and foremost pass visible light. The visible light transmittance for the two coated glass windows under test was measured and compared with a plain glass pane (4mm thick) and presented in Table 1. A handheld light meter is used to measure visible light transmittance, by averaging ten readings taken in front of and behind the glass.

TABLE I. VISIBLE Light TRANSMitTANCE

\begin{tabular}{|c|c|}
\hline Glass Types & Transmittance \\
\hline Coated Glass A & $75 \%$ \\
\hline Coated Glass B & $81 \%$ \\
\hline Plain glass & $87 \%$ \\
\hline
\end{tabular}

These results are also consistent with the transmittance for the $\mathrm{TiO} 2 / \mathrm{Ag} / \mathrm{TiO} 2$ coating shown in Figure 1. No infrared source was available to measure the transition wavelength from transmitting to reflecting for the coated glasses.

The RF propagation from $800 \mathrm{MHz}-6 \mathrm{GHz}$ was then measured and results are compared (with respect to a reference line, when no glass under test was present) in Figure 6, for an antenna separation of $5 \mathrm{~m}$. The baseline data, mentioned in Section 2.2, appears as a reference line at $0 \mathrm{~dB}$ across the range of test frequencies. Both the coated glasses show considerably reduced propagation compared with plain or no glass.

The measurements were repeated at separations of $7 \mathrm{~m}$ and $1.65 \mathrm{~m}$ (test range reduced to 1 to $2 \mathrm{GHz}$ ) and similar results were obtained.

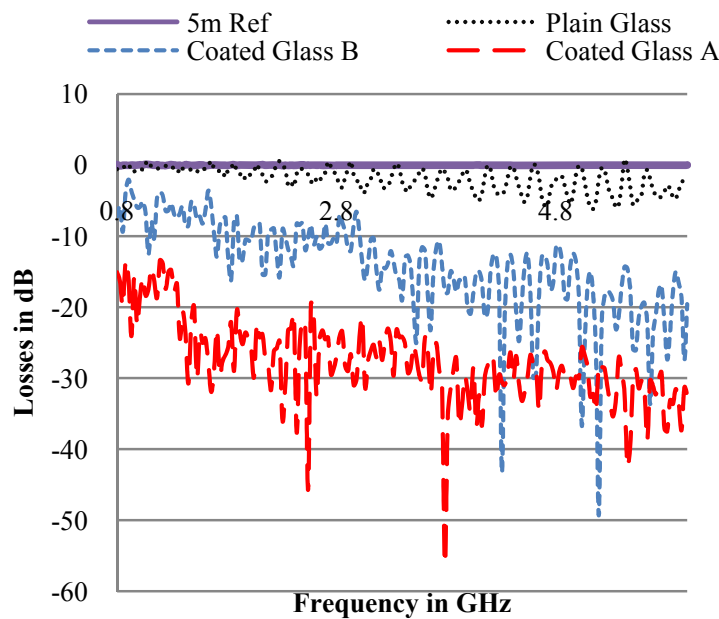

Figure6. Propagation results from large hall
The same glasses were then tested in the WIT anechoic chamber. In order to observe the far field criteria mentioned earlier, the test range was limited to $1-2 \mathrm{GHz}$. In this range, the antenna separation can be $1.65 \mathrm{~m}$, for the chosen antennas. The results are presented in Figure 7.

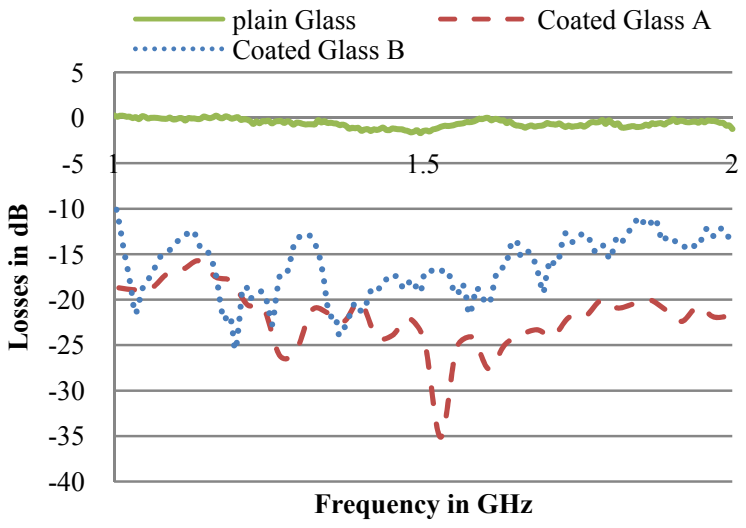

Figure7. Propagation results from anechoic chamber at $1.65 \mathrm{~m}$.

\section{ANALYSIS}

There are two analysis produced for these measurements.

\section{A. VNA Result Analisys}

The visible light transmittance results are all quite similar, especially when taking into account that Glass A is $12 \mathrm{~mm}$ total of glass (due to its large size, only $6 \mathrm{~mm}$ thick glass was feasible for each pane) and Glass B is $8 \mathrm{~mm}$ total of glass. The plain glass is $4 \mathrm{~mm}$ thick. Glass A and B are from different manufacturers and may therefore be assumed to have different coatings.

The RF propagation results for the $4 \mathrm{~mm}$ thick plain glass are always better than $-3 \mathrm{~dB}$ and correspond well with other published results. There is a gradual increase in propagation losses, as reported by, for example, NIST. For coated glass, the propagation can fall as low as $-30 \mathrm{~dB}$ and these results correspond well with previous work carried out by this group of researchers [4].

Although neither the actual coating material nor the coating thickness nor even the uniformity of deposition is known for the coated glasses under test, certain conclusions may nonetheless be drawn. Figure 1 shows a transmittance reflectance curve for a coated glass, which, from the perspective of RF propagation, may be assumed to be fairly indicative of the coating influence. (The graph may not be representative, in that the amount of transmittance/reflectance depends on material, thickness, anti-reflection material and nature of deposition).

The plasma frequency for TCs lies in the infra-red region. Below the TC plasma frequency, it acts as a conductor and reflects incident light [5]. Above the TC plasma frequency, it passes or absorbs light, acting like a dielectric. This conductorlike reflection behaviour is observed also at RF frequencies, which are much lower than those normally measured by those 
interested in TC development or those interested in window manufacture.

Both coated windows offer significantly poorer RF propagation paths compared to standard glass. The results from the anechoic chamber, in Figure 7, are consistent with the large hall measurements, in Figure 6, albeit showing even poorer propagation. The higher propagation results for the hall may be due to some small signal leakage, in spite of the foam shroud created for the receiver.

\section{B. VNA standing wave analysis}

Although the VNA is usually calibrated to the cable ends, the fluctuations in the VNA output is caused by unavoidable standing waves, due to the introduction of the MUT, whether in the large hall or the anechoic chamber. Clearly the MUT will introduce standing waves, which cannot be easily removed by calibration. It is proposed to analyse these standing wave results in the future, as a means of confirming reflection measurements, by using Fourier analysis of the VNA output. The basis for this future analysis is outlined here.

It can be shown that the VNA repeat patterns are each defined by a fundamental standing wave. For example, Figure 8 shows a close-up of the VNA output for a single $7.65 \mathrm{~m}$ cable itself. This pattern is usually calibrated out before MUT measurements.

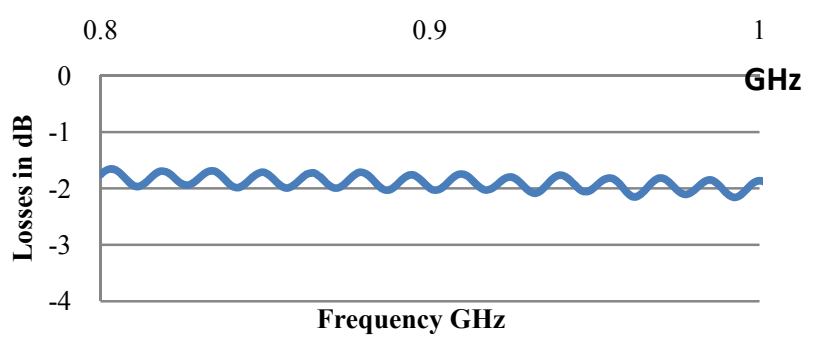

Figure8. Standing wave Chart from cable validation

The individual peak separation for constructive interference can be calculated as $15.1 \mathrm{MHz}$. To confirm the pattern is associated with the cable, if we presume the speed of light in the RF cable is lower than $c$, at $77 \%$ of the speed of light in a vacuum [6], then the fundamental standing wave wavelength is

$$
\lambda^{\prime}=\frac{\mathrm{c}^{\prime}}{\Delta \mathrm{f}}=\frac{2.31 \times 10^{8}}{15.1 \times 10^{6}}=15.29 \mathrm{~m}
$$

So the calculated cable length is $\lambda^{\prime} / 2=7.645 \mathrm{~m}$, which closely matches the actual length of $7.65 \mathrm{~m}$.

A simple example may be seen by examining the Fourier transform of VNA results shown in figure 9. The strong low frequency value may be ignored, as it is due to the VNA results being all positive (absolute values were taken).

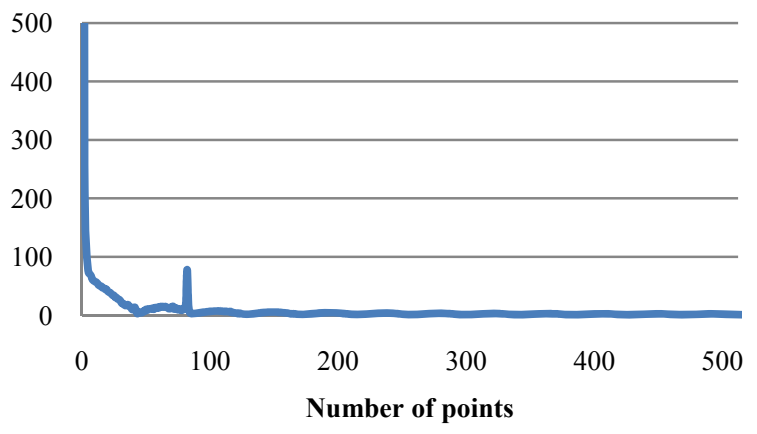

Figure9. DFT result of cable validation

The resolution of the VNA output, in this example, is

$$
\Delta \mathrm{F}=\frac{2000 \mathrm{MHz}-800 \mathrm{MHz}}{1000 \text { points }}=0.12 \mathrm{MHz}
$$

We calculate the number of samples between point $817 \mathrm{MHz}$ and $968 \mathrm{MHz}$ is 127 . There are ten periods in this range. So, the number of sample per period is close to:

$$
\begin{gathered}
\mathrm{ns}=\frac{\text { total number of points }}{\text { total periods }}=\frac{127}{10}=12.7 \\
\mathrm{f}=\frac{\mathrm{N}}{\mathrm{ns}}+1=\frac{1024}{12.7}+1 \approx 81+1=82
\end{gathered}
$$

Referring to figure 9, the relevant point is 82 . Therefore, by converting the DFT result into relevant distance, figure 10 is presented.

For figure 10 below, there is a reflection response at $\mathrm{x}=$ $7.645 \mathrm{~m}$, which is the cable length.

Analysing the more detailed output from a full MUT measurement would equally supply distance confirmation, as in the example above, but also, more importantly, a relative comparison of the impedance mismatches from various surfaces, by examining the amplitudes. If care is taken in choosing the VNA frequency range and the number of sampled points, it should be possible to even distinguish between the two different reflection coefficients in the energy efficient double glazed windows.

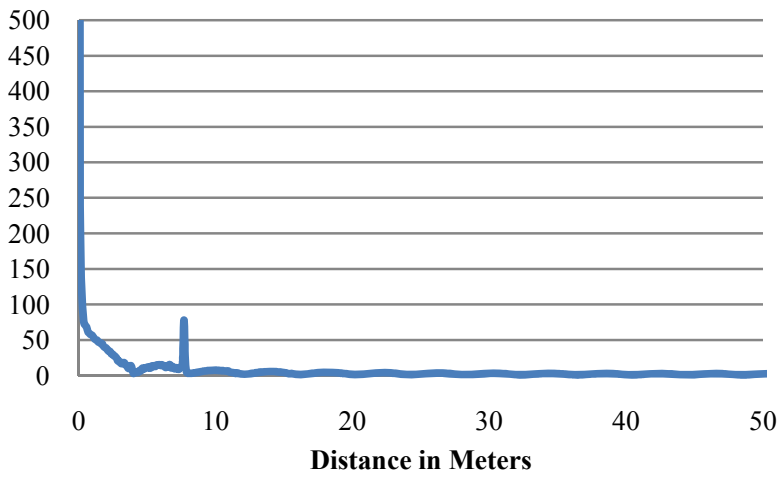

Figure10. DFT chart converted to distance response chart 


\section{CONCLUSION}

These results require further work, with more glasses and also with glass manufacturers, so that their TC information may help widen the understanding of the phenomenon.

One further question also naturally arises from these results, namely how RF signals can penetrate buildings, if the window opening is no longer, or is at least less of, an option. Other studies, for example [3] have shown the other materials in the main building fabric to be highly attenuating. The authors are consequently currently examining some traditional and some new building materials for RF propagation. Some materials offer some hope for RF propagation, particularly in the case of domestic dwellings.

On the other hand, it may be worth mentioning that RF propagation reduction is not always a disadvantage. The use of TCs on windows also means less signal leakage from the building, thereby increasing security for the inhabitants.

\section{ACKNOWLEDGMENT}

The authors would like to acknowledge support under the TSR Strand I for Yaqiang Liu and also from Bryan Hallissey in the Building Technology Department of WIT for the Total Station.

\section{REFERENCES}

[1] Bundesminsterium fuer Verkehr Bau und Stadtentwicklung, 01 October 2009

[2] Claes G. Granqvist, Transparent conductors as solar energy materials: A panoramic review. Vol.91, pp. 1529-1598, 15 October 2007.

[3] W. C. Stone, "Electromagnetic Signal Attenuation in Construction Materials." in NIST Construction Automation Program Report No. 3, October, 1997.

[4] N. Knauer, "Investigation of the Physical Effects when Electromagnetic Waves pass through various Window Types", Diploma dissertation, Fachhochschule Hannover, Germany, 2006.

[5] R.G. Gordon, "Criteria for Choosing Transparent Conductors", MRS Bulletin, vol.25, no. 8, pp. 52, August, 2000.

[6] Data Sheet of Coaxial Cable: Sucoflex 106.Huber+Suhner, Herisau, Switzerland, 2007. 